\title{
The Design of an Authoring Interface to Make eLearning Content Accessible
}

\author{
Silvia Gabrielli, Valeria Mirabella, Massimiliano Teso, and Tiziana Catarci \\ Università di Roma "La Sapienza", \\ Dipartimento di Informatica e Sistemistica, \\ via Salaria 113, 00198 Roma, Italy \\ \{gabrielli, mirabell, teso, catarci\}@dis.uniromal.it
}

\begin{abstract}
This paper presents the rationale and design process of an authoring interface that enables didactic experts to create or modify eLearning content to make it accessible by learners with special needs. The tool has been designed according to a methodological framework and a set of guidelines for eLearning accessibility previously developed by our group. A key aspect of our framework consists in helping authors to preserve the didactic quality of the eLearning experiences provided to disabled learners (in particular, visually impaired ones) beyond assuring their mere physical access to online materials. A usercentred design process has been adopted to develop a usable prototype of the authoring interface, named aLearning, that we describe below.
\end{abstract}

\section{Introduction}

Developing accessible web products and services is becoming a main commitment for many organizations, as it is strongly recommended by the law [1][2]. In the field of eLearning, in particular, accessibility assumes special importance, since large repositories of Learning Objects and resources need to be created, shared, reused to deliver courses that should fit the requirements of different learners, including also students with some kinds of impairment or disability. Relevant to this objective is an effective support of didactic authors during the preparation of eLearning material. Unfortunately, most of the authoring tools currently available provide technical advice on how to design web pages that are 'physically' accessible by users with special needs, but do not specify to authors how to transform critical contents into didactically effective alternatives for disabled learners. As part of the VICE project [I], we are experimenting with a methodology and a design process that should better support the development of high quality eLearning experiences for these types of learners. Section 2 introduces the methodological framework for eLearning accessibility that we have proposed; in Section 3 we report a brief description of aLearning, a prototype interface we have designed to make it easier for authors the deployment of our methodological framework when developing accessible eLearning contents. We conclude by envisioning some next directions of our research, to further improve the prototype developed. 


\section{Methodological Approach}

Many recommendations for the design of accessible eLearning contents have been delivered by international standard organizations, as well as private educational initiatives worldwide [6][7][8][12]. However, their level of abstraction as well as their quantity, can make it very hard for educators, who might not have prior expertise on accessibility, to effectively incorporate them into their everyday authoring practices [9]. Also, compliance of a specific web content to guidelines, as it might be assessed by the most commonly used accessibility checkers, like Bobbly [3], Lift [4], A-Prompt [5] etc., is not sufficient for eLearning material, since these tools mainly perform a syntactic assessment of web pages, but say nothing about the adequacy of any equivalent-alternative contents created, to enable effective access to this materials by disabled users during learning.

To remove this type of difficulties our work has focused, initially, on developing a methodological approach and a set of eLearning-centred guidelines for accessibility, to steer and simplify the authoring process. The method developed, that we named a 'no-frills' approach [13], is based on prompting authors to remove any content that might be considered as not essential for reaching the objectives of a learning module by learners with some level of impairment or disability. When this step has been performed and all relevant or mandatory content has been identified, the next move consists in checking if this is accessible not only from a physical point of view (like, an image described to become accessible by visually impaired learners), but also from a didactic point of view (i.e., if the content description provided does not deteriorate, in some way, the overall quality of the learning experience for a disabled learner). To develop specific guidelines or indications enabling authors to create more effective equivalent-alternative representations of critical contents, we started by analysing and selecting contents from the IEEE Learning Object Metadata (LOM) classification scheme [12], that were compliant with the criteria of format independence, such as: diagram, figure, graph and table. From the classification of learning content types provided by the CPB/WGBH National Centre for Accessible Media (NCAM) [11] we also identified and selected multimedia and math-scientific expressions. For each of these types of learning resources we analysed its impact with respect to the main typologies of impairment-disabilities that may be found in the learners' population, like visual, hearing, physical, cognitive-language ones. Each relevant match identified was then elaborated in the form of detailed guidelines for accessible eLearning content development. Due to the types of contents selected, most of the matches we have identified by now are particularly relevant to visual disabilities, however, our approach already addresses the other types of disabilities mentioned above and will be extended in the near future to include further typologies of learning resources.

The methodology and guidelines provided were initially evaluated during a formative study we conducted [10], whose main findings were fed into the design of aLearning prototype.

\section{The User-Centred Design of aLearning Prototype}

For developing aLearning prototype, beyond taking into account the guidelines for accessible authoring tools development delivered by W3C [14], we adopted a user- 
centred design approach, focused on involving several didactical experts, including also our colleagues at the university department, in providing comments and suggestions about the interface layout and functionalities.

A main motivation of our work was to develop a domain specific accessibility tool for the field of eLearning, that was intuitive to use even for authors without specific expertise on accessibility and/or that might not have a technical kind of background. The tool is meant to facilitate their creation of more accessible and usable eLearning contents, by making automatic or semiautomatic any technical step of the methodology that would not benefit from human intervention or expertise (such as the identification of critical contents within the eLearning material), but prompting, informing and capitalizing on authors' knowledge and decision making when addressing the non technical steps of the method (e.g., creating an alternative-equivalent version of the content). A representation of our methodological approach and guidelines by means of task analysis was carried out, leading to subsequent phases of low-mid tech prototyping of the interface features required. We also applied walkthrough evaluation techniques to assure consistency of the prototype and to make its exploration as familiar and intuitive as possible from a user perspective.

\subsection{Overview of aLearning Prototype}

Currently, aLearning interface mainly supports authors in:

- automatically identifying and marking any critical and inaccessible content within the eLearning material. Differently from other existing accessibility tools, it considers didactically relevant categories of contents (such as diagrams, figures, graphs, etc.), instead of web domain ones (like framesets, scripts, links, etc).

- Enabling the user to select and start repairing that content (e.g., a graph) by choosing among three alternative modalities: i) directly clicking on the critical content as marked within the course pages, ii) selecting that content from the list of learning resource categories reported on a frame window, iii) starting a stepby-step repairing procedure for the whole sequence of inaccessible contents identified. These different modalities are expected to provide authors more flexibility on how to complete the repairing process (also in terms of its timescale), differently from the file-by-file repairing process enabled by other tools.

- Explaining to the user why a specific content is inaccessible, prompting the user to classify it as optional or mandatory for the objectives of the course, supporting different ways of creating alternative versions of the content if it is mandatory, providing links to more detailed information (guidelines and examples) on how to create an appropriate alternative representation of it.

Our efforts are also addressed towards refining and improving the quality of usersystem communication implemented so far, to speed up not only any repairing process performed by the user, but also authors' acquisition of expertise on accessibility by means of use and navigation through the prototype functionalities.

The tool is being developed in Java, we expect to integrate it with a content management system which receives XML pages as input and transforms them into XHTML. 


\section{Conclusions and Future Work}

We are planning a series of empirical user studies, involving different authors working on different topics and types of Learning Objects, to evaluate the effectiveness, quality of performance, and user satisfaction during the authoring of accessible eLearning materials supported by our prototype. In addition, we are investigating if other accessibility functionalities should be added, like for instance, authors' specification of accessibility metadata for the materials they have created or modified.

We expect that all these future steps will enable us to improve our design outcomes in terms of their usability and level of contribution to the accessibility research field.

Acknowledgments. This work is supported by the VICE project [Comunità Virtuali per la Formazione, CNR-MIUR, Italy].

\section{References}

1. Section 508 of the Rehabilitation Act; see http://www.section508.gov

2. Law 9 January 2004, n. 4; http://www.pubbliaccesso.it/normative/legge_20040109_n4.htm

3. Bobby, Watchfire Corporation; see http://bobby.watchfire.com/

4. LiFT, http://www.usablenet.com/

5. A-Prompt, University of Toronto, http://aprompt.snow.utoronto.ca/

6. Web Content Accessibility Guidelines 2.0, W3C Recommendations, Working Draft 19 November 2004, http://www.w3.org/TR/WCAG20

7. Advanced Distributed Learning, http://www.adlnet.org/

8. IMS Guidelines for Developing Accessible Learning Applications, Version 1.0, White Paper 2002, http://www.imsproject.org/accessibility

9. Bennett, S., Hewitt, J., Kraithman, D., Britton, C.: Making Chalk and Talk Accessible. Proc. of the ACM Conf. on Universal Usability (2003) 119-125

10. Gabrielli, S., Mirabella, V., Kimani, S., Catarci, T.: Steering the Development of Accessible e-Learning Content. In Proc. of the 3rd ECEL Conf. (2004) Paris (F) 517-526

11. Freed, G., Rothberg M., Wlodkowski T. (Eds.): Making Educational Software and Web Sites Accessible. Design Guidelines Including Math and Science Solutions (2003), http://ncam.wgbh.org/cdrom/guideline

12. Hodgins, W. \& Duval, E. (Eds.): IEEE LTSC Learning Object Meta-data LOM_1484_12_1_v1_Final_Draft, (2002), http://ltsc.ieee.org/wg12/files/

13. Mirabella, V., Kimani, S., Gabrielli, S., Catarci, T.: Accessible e-Learning Material: A NoFrills Avenue for Didactical Experts, The New Review of Hypermedia and Multimedia, Vol.10 N.2 (Dec. 2004) 1-16

14. Treviranus, J., McCathieNevile, C., Jacobs, I., Richards, J. (Eds.): Authoring Tool Accessibility Guidelines 1.0. W3C Recommendation (2000), http://www.w3.org/TR/ATAG10/ 